\title{
Wiener Number of Some Subgraphs in Archimedean Tilings
}

\author{
Yunjing Pan \\ Cheng Yi College, Jimei University, Xiamen, China \\ Email: 382194460@qq.com
}

How to cite this paper: Pan, Y.J. (2021) Wiener Number of Some Subgraphs in Archimedean Tilings. Applied Mathematics, 12, 939-946.

https://doi.org/10.4236/am.2021.1211062

Received: September 28, 2021

Accepted: November 6, 2021

Published: November 9, 2021

Copyright $\odot 2021$ by author(s) and Scientific Research Publishing Inc. This work is licensed under the Creative Commons Attribution International License (CC BY 4.0).

http://creativecommons.org/licenses/by/4.0/

\begin{abstract}
In this paper, we deduce Wiener number of some connected subgraphs in tilings $(4,4,4,4)$ and $(4,6,12)$, which are in Archimedean tilings. And compute their average distance.
\end{abstract}

\section{Keywords}

Archimedean Tiling, Wiener Number, Binary Hamming Graph, Average Distance

\section{Introduction}

One of the molecular-graph-based quantity $W$, introduced by Harold Wiener in 1947 [1], is nowadays known as the name Wiener index or Wiener number. For a connected graph $G$, let $V(G)$ denote the set of vertices and $E(G)$ the set of edges. Then the Wiener number of $G$, denoted by $W(G)$, is defined by:

$$
W(G)=\sum_{u, v \subseteq V(G)} d(u, v \mid G)
$$

where $d(u, v \mid G)$ is the distance between vertices of $u$ and $v$, and the summation goes over all vertices in $V(G)$.

It is found that many physical and chemical properties that depend primarily on the compactness and the extent of branching are usually well correlated with W. And Wiener number has a lot of applications in different fields [2] [3] [4] [5].

From the definition of Wiener number, it is easy to find that the calculation of Wiener number in a lot of graphs is rather complicated. As a result, people research on the method of computing the Wiener number of graphs: such as the Wiener number of the hexagonal system; extremal tree on Wiener number and so on [6] [7] [8]. In 1947, Wiener already gave a much more convenient formula 
to compute the Wiener number of trees: we denote a tree by $T, n_{1}(e)$ and $n_{2}(e)$ are the number of vertices of Tlying on the two sides of the edge $e$, then:

$$
W(T)=\sum_{e} n_{1}(e) n_{2}(e)
$$

where the summation goes over all edges of $T$.

For a connected graph $G$, the average distance of $G$ is another index, which depends on the Wiener number. The average distance of $G$ is denoted by $W(G)$, and is defined by:

$$
\bar{W}(G)=\frac{W(G)}{C_{n}^{2}},
$$

where $n$ is the number of vertices of $G$.

A plane tiling $T=\left\{T_{1}, T_{2}, \cdots\right\}$ is a countable family of closed sets which covers the plane without gaps and overlaps, where $T_{1}, T_{2}, \cdots$ are known as tiles of $T$ [9].

In a tiling, a vertex is of type $n_{1} n_{2} \cdots n_{r}$ if it is surrounded in cyclic order by regular n-gons of order $n_{1}, n_{2}, \cdots$ and $n_{r}$.

There exist precise 11 distinct types of edge-to-edge tilings by regular polygons such that all vertices of the tiling are of the same type. These 11 types of tilings are usually called Archimedean tilings [9].

They are: $(3,3,3,3,3,3),(4,4,4,4),(6,6,6),(4,4,3,3,3),(4,8,8),(4,3,4,3$, $3),(6,3,6,3),(6,3,3,3,3),(4,6,12),(12,3,12),(6,4,3,4)$ (Figure 1).

In this paper, we shall restrict attention to Archimedean tilings. And the subgraphs in Archimedean tilings are simply-connected graphs.

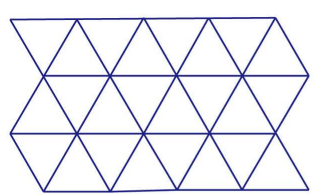

$(3,3,3,3,3,3)$

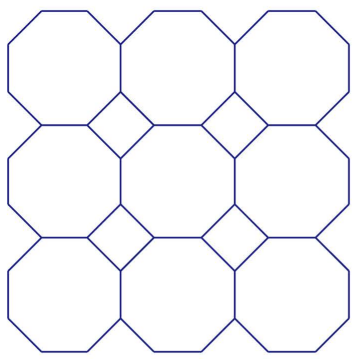

$(8,8,4)$

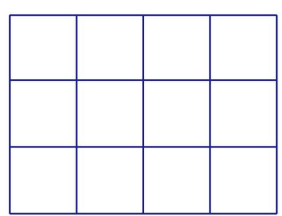

$(4,4,4,4)$

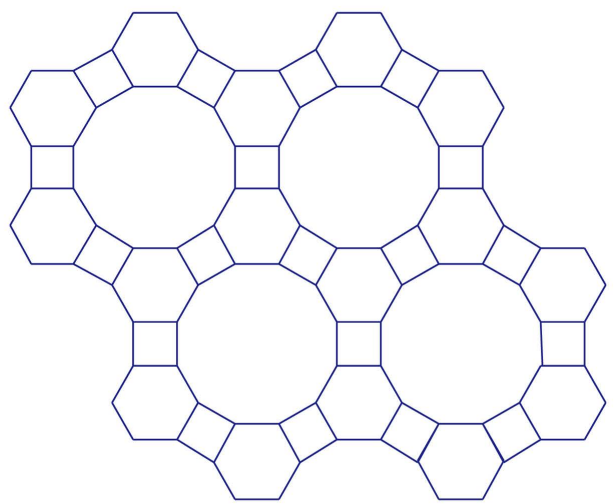

$(4,6,12)$

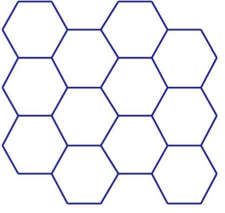

$(6,6,6)$

Figure 1. Some archimedean tilings. 
$\Sigma$ be a finite a alphabet and let $w_{1}$ and $w_{2}$ be words of equal length over

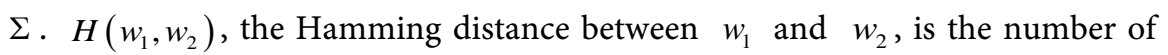
positions in which $w_{1}$ and $w_{2}$ differ. For the graph $G$, if each vertex $v \in V(G)$ can be labeled by a word $l(v)$ of fixed length, such that $H(l(u), l(v))=d(u, v)$ for all $u, v \in V(G)$, we call it Hamming graph. In particular, if $\Sigma=\{0,1\}$, we call $G$ a binary Hamming graph.

$G$ is a subgraph of one of tilings $(4,4,4,4),(6,6,6),(4,8,8),(4,6,12)$. An elementary cut segment $C$ is a straight line segment drawn orthogonal to some edges; starting from the perimeter and ending at the perimeter; and touching the perimeter only twice; deleting the edges which orthogonal to $C$, there are exactly two connected components (Figure 2).

About fifty years later, Sandi Klavzar, Ivan Gutman, Bojan Mohar found a similarly convenient way to calculate the Wiener number of binary Hamming graphs [10]. Later, they proved that all connected subgraphs of tiling $(6,6,6)$ are binary Hamming graphs, and they computed the Wiener number of some benzenoid hydrocarbons [11]. Furthermore, only four tilings in Archimedean tilings, which are $(4,4,4,4),(6,6,6),(4,8,8)$ and $(4,6,12)$ tilings, all their connected subgraphs are binary Hamming graphs that are proved. And follow the way mentioned in [10], a convenient way to compute the Wiener number of the subgraphs of tilings $(4,4,4,4),(6,6,6),(4,8,8)$ and $(4,6,12)$ are proved [12], which is called the elementary cut method. Let $G$ be a binary Hamming graph on $n$ vertices and with $k$ elementary cut segments. For $i=1, \cdots, k$, let $n_{i}$ be the number of vertices of $G$ in one of the components of the graph obtained from $G$ by removing the $i$ th elementary cut.

Then:

$$
W(G)=\sum_{i=1}^{k} n_{i}\left(n-n_{i}\right),
$$

The elementary cut method is similar to the formula to compute the Wiener number of trees.

In this paper, we deduce the Wiener number of some interesting subgraphs in tilings $(4,4,4,4)$ and $(4,6,12)$, and compute their average distance.

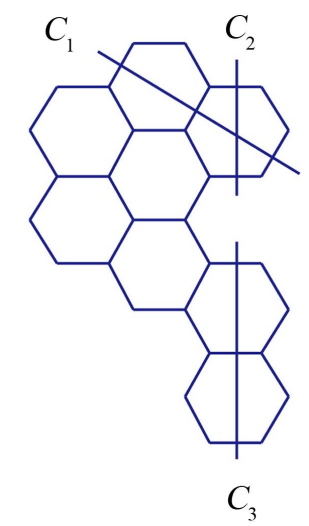

Figure 2. Elementary cut segment. 


\section{The Wiener Number of Some Subgraphs in Tiling} $(4,4,4,4)$ and $(4,6,12)$

1) Aztec diamond graph. A Aztec Diamond of order $n$, denoted by $H_{n}$, is a plane graph consisted by squares of length 1 (Figure 3 and Figure 4). Many work has been done about Aztec Diamond graph, such as the problem of perfect matching and independent set of it [10] [11]. Now we consider the Wiener number of Aztec Diamond.

In Figure 4, we give the horizontal elementary cut segments of $H_{n}$. There exist one additional groups of symmetry-equivalent elementary cut segments, obtained by rotating the former group by $+90^{\circ}$. Therefore, if one applies Equation (2) to only the horizontal elementary cut segments, the result will be just one second of the Wiener number of $H_{n}$.
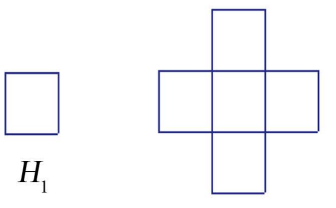

$\mathrm{H}_{2}$

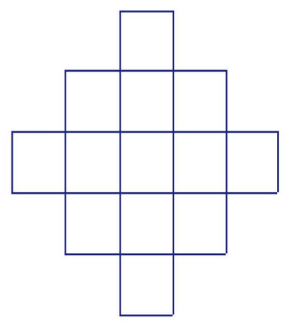

$\mathrm{H}_{3}$

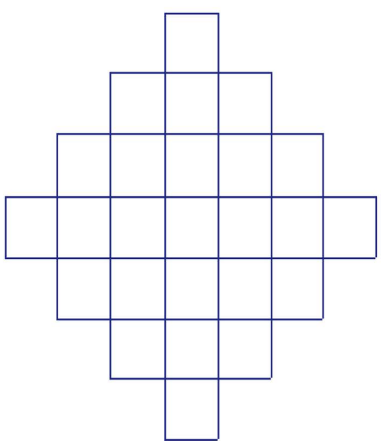

$\mathrm{H}_{4}$

Figure 3. Aztec diamond graph $H_{1}, H_{2}, H_{3}, H_{4}$.

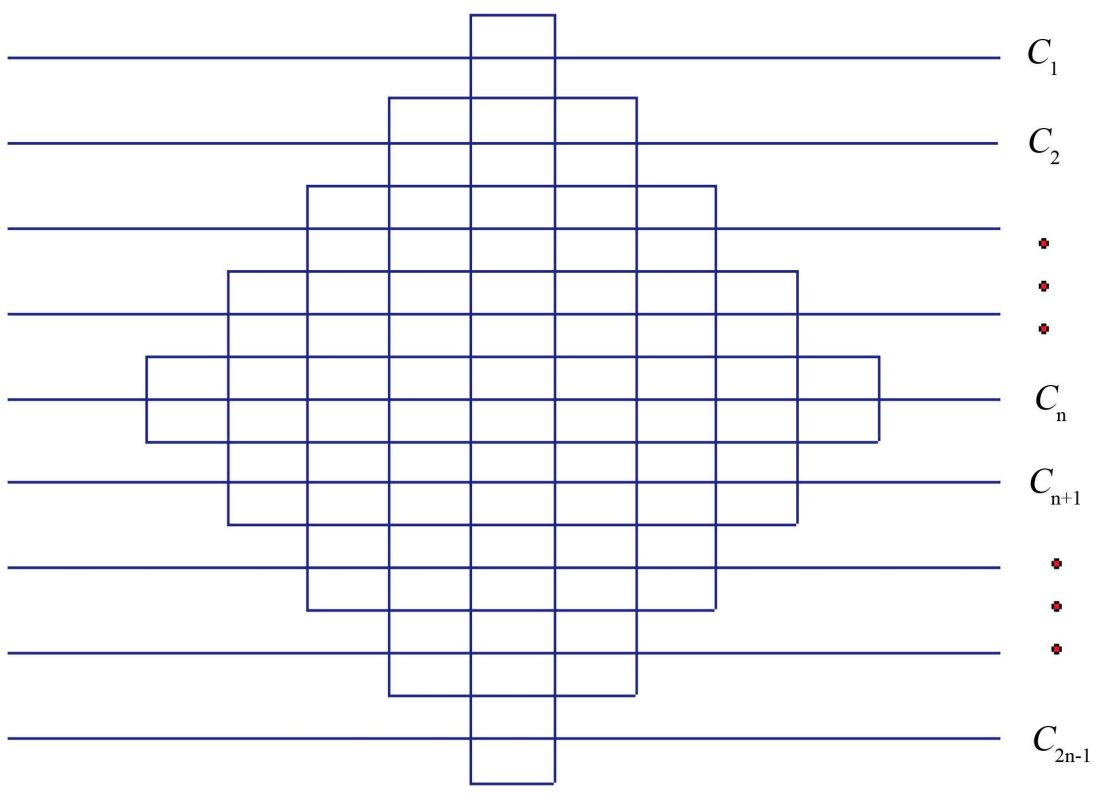

$H_{n}$

Figure 4. Aztec diamond graph $H_{n}$. 
First, we calculate the number of $H_{n}$. It is easy to get:

$$
\begin{aligned}
& n\left(H_{n+1}\right)-n\left(H_{n}\right)=4 n+4, \\
& n\left(H_{n}\right)-n\left(H_{n-1}\right)=4(n-1)+4, \\
& n\left(H_{n-1}\right)-n\left(H_{n-2}\right)=4(n-2)+4, \\
& \vdots \\
& n\left(H_{2}\right)-n\left(H_{1}\right)=4+4,
\end{aligned}
$$

To sum up the equations above, we get:

$$
n\left(H_{n+1}\right)-n\left(H_{1}\right)=4(1+2+3+\cdots+n)+4 n=2 n^{2}+6 n,
$$

That is: $n\left(H_{n}\right)=2 n^{2}+2 n$.

For arbitrary horizontal elementary cut segment $C_{i}(i=1,2, \cdots, n)$, $n\left(G_{C_{i}}^{0}\right)=2+4+\cdots+2 i=i(i+1)$.

Denote $C_{i}(i=1, \cdots, 2 n-1)$ contribute to the Wiener number of $G$ is $W_{1}$, then we have:

$$
W_{1}=\sum_{C_{i}} n\left(G_{C_{i}}^{0}\right) n\left(G_{C_{i}}^{1}\right)=2 \sum_{i=1}^{n-1}(i+1) i\left[2 n^{2}+2 n-i(i+1)\right]+n^{2}(n+1)^{2},
$$

Simplify the equation above:

$$
W\left(H_{n}\right)=2 W_{1}=(2 / 15)\left(14 n^{5}+35 n^{4}+20 n^{3}-5 n^{2}-4 n\right) .
$$

Furthermore, we get $\bar{W}(H(n))=\frac{(2 / 15)\left(14 n^{5}+35 n^{4}+20 n^{3}-5 n^{2}-4 n\right)}{C_{2 n^{2}+2 n}^{2}}$. When $n$ gets large enough, $\bar{W}(H(n))$ approximates to $(14 / 15) n$.

\section{2) Zig-zag polyomino chain}

As illustrate in Figure 5, it is a zig-zag chain $G$ which contains $n$ squares. Notice that $n$ is even, suppose that $n=2 m$. It is easy to get $n(G)=2 n+2$.

In Figure 5 are indicated two groups of elementary cut segments: one group is horizontal elementary cut segments $C_{i}(i=1, \cdots, m)$ (labeling from up to down), another group is $A_{j}(j=1, \cdots, m+1)$ (labeling from left to right).

Denote $C_{i}(i=1, \cdots, m)$ contribute to the Wiener number of $G$ is $W_{1}$, $A_{j}(j=1, \cdots, m+1)$ contribute to the Wiener number of $G$ is $W_{2}$.

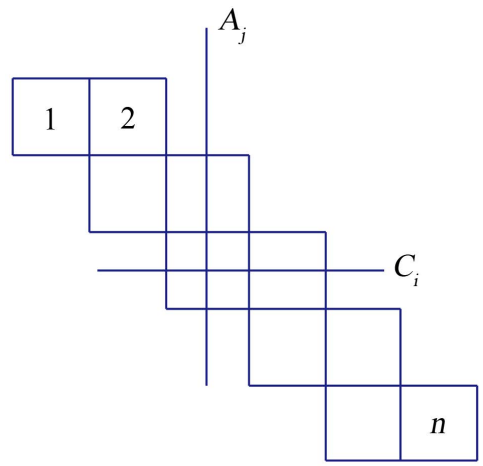

G

Figure 5. Zig-zag polyomino chain. 


$$
\begin{gathered}
W_{1}=\sum_{C_{i}} n\left(G_{C_{i}}^{0}\right) n\left(G_{C_{i}}^{1}\right)=6(2 n-1)+\sum_{k=1}^{m-2}(3+4 k)(2 n+2-3-4 k), \\
W_{2}=\sum_{A_{j}} n\left(G_{A_{j}}^{0}\right) n\left(G_{A_{j}}^{1}\right)=8 n+10(2 n-3)+\sum_{k=1}^{m-3}(5+4 k)(2 n+2-5-4 k) .
\end{gathered}
$$

Simplify the two equations above:

$$
\begin{gathered}
W_{1}=(1 / 3) n^{3}+n^{2}+(7 / 6) n, \\
W_{2}=(1 / 3) n^{3}+n^{2}+(31 / 6) n-1 .
\end{gathered}
$$

Then we get:

$$
W(G)=W_{1}+W_{2}=(2 / 3) n^{3}+2 n^{2}+(19 / 3) n-1 .
$$

Furthermore, we get $\bar{W}(G)=\frac{(2 / 3) n^{3}+2 n^{2}+(19 / 3) n-1}{C_{2 n+2}^{2}}$. When $n$ gets large enough, $\bar{W}(H(n))$ approximates to $(1 / 12) n$.

3) At last, we give a linear subgraph $G$ of tiling $(4,6,12)$, as illustrated in Figure 6. It contains $n$ regular dodecagons.

It is easy to calculate: when $n=1, W(G)=2304$; when $n=2$, $W(G)=11556$; when $n=3, W(G)=29052$; when $n=4, W(G)=70020$. Next, we compute the Wiener number of $G$ when $n \geq 5$.

It is easy to get the number of vertices of $G$ is:

$$
n(G)=36+24(n-1)=24 n+12 \text {. }
$$

There are four groups elementary cut segments: the first is horizontal elementary cut segment $C, n\left(G_{C}^{0}\right)=12 n+6$, denote $C$ contribute to $W(G)$ is $W_{1}$; the second group is $H_{k}(k=1,2, \cdots, 2 n+1), n\left(G_{H_{k}}^{0}\right)=6+12 k$, denote this group contribute to $W(G)$ is $W_{2}$; the third group is $A_{j}$ and $A_{j}^{\prime}$ $(j=1,2, \cdots, n)$, here $A_{j}^{\prime}$ can be obtained by rotating $A_{j}$ a certain angle, so $A_{j}$ and $A_{j}^{\prime} \quad(j=1,2, \cdots, n)$ contribute to $W(G)$ are the same, denote $A_{j}(j=1,2, \cdots, n)$ contribute to $W(G)$ is $W_{3}$; the fourth group is $C_{i}$ and $C_{i}^{\prime}$ $(i=1,2, \cdots, n+2)$, here $C_{i}^{\prime}$ can be obtained by rotating $C_{i}$ a certain angle, so $C_{i}$ and $C_{i}^{\prime}(i=1,2, \cdots, n+2)$ contribute to $W(G)$ are the same, denote $C_{i}$ $(i=1,2, \cdots, n+2)$ contribute to $W(G)$ is $W_{4}$.

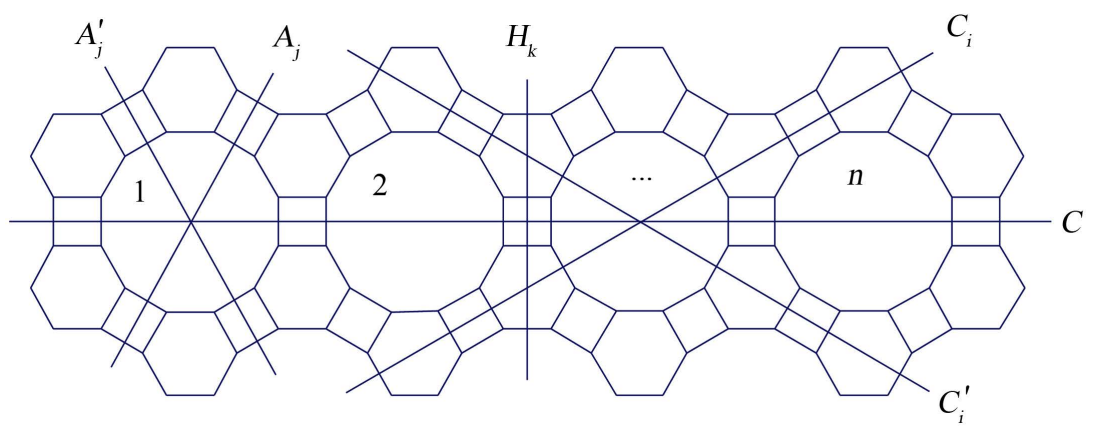

G

Figure 6. A linear subgraph $G$ of tiling $(4,6,12)$. 
By calculating:

$$
\begin{gathered}
W_{1}=(12 n+6)^{2}=144 n^{2}+144 n+36, \\
W_{2}=\sum_{H_{k}} n\left(G_{H_{k}}^{0}\right) n\left(G_{H_{k}}^{1}\right) \\
=6(24 n+12-6)+\sum_{k=0}^{2 n}(6+12 k)(24 n+12-6-12 k) \\
=192 n^{3}+288 n^{2}+168 n+36, \\
W_{3}=\sum_{A_{j}} n\left(G_{A_{j}}^{0}\right) n\left(G_{A_{j}}^{1}\right) \\
=\sum_{j=0}^{n-1}(18+24 j)(24 n+12-18-24 j) \\
=192 n^{3}+288 n^{2}+168 n, \\
W_{4}=\sum_{C_{i}} n\left(G_{C_{i}}^{0}\right) n\left(G_{C_{i}}^{1}\right) \\
=12(24 n+12-6)+42(24 n+12-21)+84(24 n+12-42) \\
+\sum_{i=1}^{n-4}(42+24 i)(24 n+12-42-24 i) \\
=96 n^{3}+144 n^{2}+516 n-90 .
\end{gathered}
$$

Therefore the Wiener number of $G$ is:

$$
W(G)=W_{1}+W_{2}+2 W_{3}+2 W_{4}=576 n^{3}+1008 n^{2}+1512 n-108 .
$$

Furthermore, we get $\bar{W}(G)=\frac{576 n^{3}+1008 n^{2}+1512 n-108}{C_{24 n+12}^{2}}$. When $n$ gets large enough, $\bar{W}(H(n))$ approximates to $2 n$.

\section{Conclusion Remark}

In this paper, we deduce the Wiener number of Aztec Diamond graph, zig-zag polyomino chain in tiling $(4,4,4,4)$ and a linear subgraph in tiling $(4,6,12)$, and find their average distance.

\section{Funding}

This work was supported by the Fujian Provincial Department of Education Young and Middle-aged Teacher Education Research Project (No. JAT191154).

\section{Conflicts of Interest}

The author declares no conflicts of interest regarding the publication of this paper.

\section{References}

[1] Wiener, H. (1947) Structural Determination of Paraffin Boiling Points. Journal of the American Chemical Society, 69, 17-20. https://doi.org/10.1021/ja01193a005

[2] Banerjee, S., Nath, D. and Chaudhuri, B.K. (1982) Phenomenological Theory of the 
Isostructural Phase Transition in $\mathrm{H}$-Bonded $\mathrm{SnCl}_{2} \cdot\left(\mathrm{H}_{2} \mathrm{O}\right)_{2}$ and $\mathrm{SnCl}_{2} \cdot\left(\mathrm{H}_{2} \mathrm{O}\right)_{x}\left(\mathrm{D}_{2} \mathrm{O}\right)_{2-x}$ with a Pseudospin Model. Physical Review B, 25, 1883. https://doi.org/10.1103/PhysRevB.25.1883

[3] Basak, S.C., Restrepo, G. and Villaveces, J.L. (2014) Advances in Mathematical Chemistry and Applications. Hentham Science e-book, Vol. 1.

[4] Bonchev, D. (2002) The Wiener Number, Some Applications and New Developments. In: Topology in Chemistry: Discrete Mathematics of Molecules. Woodhead Publishing, Chichester, 58-88. https://doi.org/10.1016/B978-1-898563-76-1.50008-1

[5] Diudea, M.V., Gutman, I. and Jantschi, L. (2001) Molecular Topology. Nova, Huntington.

[6] Dobrynin, A.A., Entringer, R. and Gutman, I. (2001) Wiener Index of Trees: Theory and Applications. Acta Applicandae Mathematica, 66, 211-249. https://doi.org/10.1023/A:1010767517079

[7] Shiu, W.C. and Lam, P.C.B. (1997) Wiener Numbers of Some Pericondensed Benzenoid Molecule Systems. Congrseeus Numerantium, 126, 113-124.

[8] Gutman, I., Yeh, Y.N., Lee, S.L. and Luo, Y.L. (1993) Some Recent Results in the Theory of the Wiener Number. Indian Journal of Chemistry, 32A, 651-661.

[9] Ng Lay Ling (2004) Tilings and Patterns. Honours Project, Department of Mathematics, National Univercity of Singapore.

[10] Klavzar, S., Gutman, I. and Mohar, B. (1995) Labeling of Benzenoid Systems which Reflects the Vertex-Distance Relations. Journal of Chemical Information and Computer Science, 35, 590-593. https://doi.org/10.1021/ci00025a030

[11] Gutman, I. and Klavzar, S. (1996) A Method for Calculating Wiener Numbers of Benzenoid Hydrocarbons. Models in Chemistry, 133, 389-399.

[12] Pan, Y.J., Xie, M.F. and Zhang, F.J. (2018) Partial Cubes and Archimedean Tilings. Acta Mathematicae Applicatae Sinica., 34, 782-791. https://doi.org/10.1007/s10255-018-0788-0 